\title{
EFEITOS DO MÉTODO OCLUSÃO VASCULAR SOBRE AS ADAPTAÇÕES TENDÍNEAS
}

As respostas adaptativas do tendão ao exercício físico representam uma área de grande interesse. Considerando que o movimento humano ocorre devido a força criada pelos músculos e transmitida aos ossos via tendão, o efeito do método oclusão vascular sobre as adaptações tendíneas é também um tema que deve ser explorado em estudos futuros, principalmente associado a reabilitação.

O tecido do tendão consiste predominantemente de fibras colágenas do tipo I e III e o papel dos fatores de crescimento como GH e IGF-1 que influenciam o aumento da síntese de colágenos, podem promover um fator protetivo contrarrupturas (KJÆR et al., 2009). Logo, a resposta hormonal promovida pelo método oclusão vascular é de grande interesse.

O treinamento resistido per se e a manipulação de suas variáveis, podem influenciar a resposta hormonal durante o treinamento. Além disso, as respostas hormonais induzidas quando o treinamento resistido é realizado com a oclusão vascular podem influenciar também as adaptaçōes tendíneas.

A secreção do $\mathrm{GH}$ é estimulada por um ambiente intramuscular ácido e a mesma via que estimula uma maior resposta do sistema nervoso autonômico simpático através do metaborreceptores e mecanoceptores é também a mesma via que estimula maior secreção de GH pela glândula pituitária anterior quando o pH está baixo (LOENNEKE; WILSON; WILSON, 2010).

Kubo et al. (2010) demonstraram pela primeira vez que as adaptaçóes tendíneas não ocorrem similarmente aos aumentos de força muscular, hipertrofia e ganhos neurais. As adaptações tendíneas (stiffness) em seu estudo somente atingiram 
significância estatística após 3 meses de treinamento. No entanto, permaneceram acima dos valores basais após 3 meses de destreinamento.

Parece também que existe uma dose resposta para adaptações tendíneas e Grosset et al. (GROSSET et al., 2014) verificaram que em idosos com idade entre 68 e 74 anos separados em dois grupos, somente o grupo que treinou com uma intensidade de $80 \%$ de 1RM teve adaptações tendíneas quando comparado ao grupo de baixa intensidade (40\% de $1 \mathrm{RM}$ ) após 12 semanas de treinamento. Concluindo que o treinamento de baixa intensidade não gera adaptações benéficas aos tendões em indivíduos idosos.

Com isso, Kubo et al. (2006) demonstraram pela primeira vez que após 12 semanas de treinamento resistido e com oclusão vascular realizado com baixa intensidade (20\% de 1RM) não promoveu alterações sobre as propriedades tendíneas quando comparado com o treinamento de alta intensidade ( $80 \%$ de $1 \mathrm{RM})$. Sugerindo que somente o estresse mecânico e não metabólico é que contribuirá para as adaptações tendíneas.

O conhecimento dessa adaptação é muito importante principalmente nos processos de reabilitação. Associar a grande resposta hormonal durante o treinamento com oclusão vascular (SHIMIZU et al., 2016; TAKANO et al., 2005) a uma melhor adaptação tendínea ainda precisa de maior esclarecimento em futuras pesquisas científicas.

\section{REFERÊNCIAS BIBLIOGRÁFICAS}

GROSSET, Jean-Francois et al. Influence of exercise intensity on training-induced tendon mechanical properties changes in older individuals. Age, v. 36, n. 3, p. 9657, 2014.

KJÆR, Michael et al. From mechanical loading to collagen synthesis, structural changes and function in human tendon. Scandinavian journal of medicine \& science in sports, v. 19, n. 4, p. 500-510, 2009.

KUBO, Keitaro et al. Effects of low-load resistance training with vascular occlusion on the mechanical properties of muscle and tendon. Journal of applied biomechanics, v. 22, n. 2, p. 112-119, 2006.

KUBO, Keitaro et al. Time course of changes in muscle and tendon properties during strength training and detraining. The Journal of Strength \& Conditioning Research, v. 24, n. 2, p. 322-331, 2010.

LOENNEKE, J. P.; WILSON, G. J.; WILSON, J. M. A mechanistic approach to blood flow occlusion. International journal of sports medicine, v. 31, n. 01, p. 1-4, 2010.

SHIMIZU, Ryosuke et al. Low-intensity resistance training with blood flow restriction improves vascular endothelial function and peripheral blood circulation in healthy elderly people. European journal of applied physiology, v. 116, n. 4, p. 749-757, 2016.

TAKANO, H. et al. Effects of low-intensity "KAATSU” resistance exercise on hemodynamic and growth hormone responses. International Journal of KAATSU Training Research, v. 1, n. 1, p. 13-18, 2005. 